\title{
32 \\ Reducing the cost of Remote Procedure Call
}

\author{
A. L. Ibbetson, P. F. Linington, I. A. Penny, \\ A. B. Smith and G. E. W. Tripp \\ University of Kent, Canterbury, Kent, CT2 7NF, England. \\ Tel: +44 1227 827535, Fax: +44 1227762811 , \\ Email: I.A.Penny@ukc.ac.uk
}

\begin{abstract}
An RPC implementation is described which uses a small network of Transputers as a parallel front end processor to reduce communications delays. Parallel processing allows different aspects of the protocol support and parameter marshalling to be overlapped. Particular attention is paid to issues which affect the performance of the system. Some comparisons are drawn with the ANSAware distributed systems platform.
\end{abstract}

\section{INTRODUCTION AND BACKGROUND}

Modern distributed systems are frequently based on a Remote Procedure Call (RPC) communication model. This model is easy to integrate with a wide range of programming languages, and is increasingly being used as the preferred means of access in supporting distribution. It is a natural choice to realize modern distributed architectures, such as that standardized by the ISO and ITU for Open Distributed Processing [ISO, 1995] [Linington, 1995], There is an increasing number of products based on this style of communication. The ANSAware infrastructure, which can be taken as representative of the modern generation of open platforms [ANSA, 1989], is the basis for the study reported here, but the results are equally applicable to, for example, a variety of CORBA implementations [OMG, 1991].

The performance of the distributed applications produced using such platforms depends on the time taken for each remote procedure call interaction. This time is dominated by the latency (end-to-end delay) of the chain of communication activities supporting the interaction, and thus becomes the most important aspect of communications Quality of Service for such systems. It is generally dominated by software costs, rather than by the data transmission required, and so its reduction must be treated as a system problem.

Much work has been done to investigate operating system structures which lead to efficient RPC interactions. Classic examples are Firefly [Schroeder, 1990], Amoeba [van Renesse, 1988] and Chorus [Rozier, 1986]. However, they have had limited commercial take up, because of the huge investment in application software and staff resources represented by the more traditional operating systems such as Unix; users have come to expect the rich management structure that such systems now provide. Therefore this paper considers use of an established operating system supported by a parallel processing network front end.

Several researchers have considered the use of parallel processors to support a multilayer communications architecture, but studies have shown that mapping a stack of protocols directly 
onto a corresponding stack of communicating processes gives rather poor latency for only moderate improvements in bulk throughput. This approach is quite unsuited to the support of RPC based systems where latency is critical.

The architecture proposed here is based instead on optimistic concurrency. The idea is that priority is given to identifying the major tasks (such as the analysis of various protocol headers) and then passing these tasks to individual processors for parallel execution. The results are passed to a coordinating process which uses the results if and only if they were all successful. In most cases, all the activities will succeed, and therefore the various layers of decoding and transformation will have progressed in parallel. If one of the activities fails or identifies an unusual situation, the remainder are rolled back and may be restarted in a modified form if necessary.

This parallelism should include the local operating system actions needed to locate the receiving user address space because this activity has a large effect on performance. Priority is therefore given to extracting a provisional service identity and passing it to the host operating system, which can then activate the target process. This can then request the RPC payload. If protocol analysis subsequently fails, the user request receives a reply indicating "false alarm" and terminates.

The time needed to perform an RPC increases rapidly as the RPC payload increases. Marshalling and unmarshalling rapidly become the major costs for large parameter sets, particularly in open platforms where the basic data items may need to be converted to or from the format required by the host machine architecture, or if a heavyweight general purpose encoding such as ASN.1 is used.

Savings could be made if the marshalling and unmarshalling was performed outside the application. However there is then a need for additional communication with the front end to convey the signatures of newly bound RPC contexts and to free resources after use. In conventional architectures it is difficult to do much about the marshalling cost because the control information needed is contained within the user address space on the host. The design described here passes the necessary template to the communication front end to allow the work to be carried on outside the user's address space.

Figure 1 below indicates the sequence of events during reception of a normal RPC invocation. The situation during initiation is broadly similar. The different protocol components are processed in sequence, with an delay midway through the activity while the target user process is identified and cross address space transfer performed. Note that each part of this processing is completed before the next can begin, so that there is no gain from parallelism. Indeed, a multiprocessor implementation would probably be slower, because of the interprocessor communication overhead.

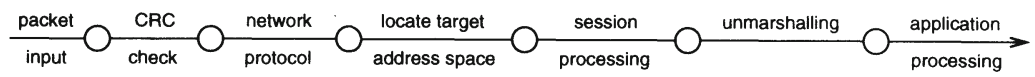

Figure 1 Serial processing of an RPC invocation

Figure 2 indicates the situation if each of these steps can be initiated in parallel, extracting the necessary information from the received packet on the assumption that it has the commonest packet format and raises no exceptions.

There will, of course, be some housekeeping activity in each of the parallel processes after the payload has been transferred to the host, as each process must accept a commit request from the 


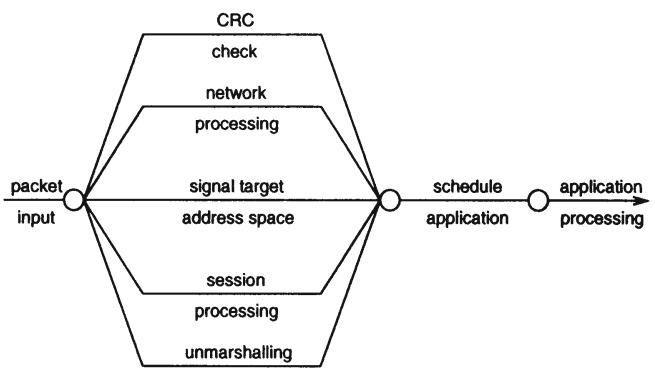

Figure 2 Parallel processing of an RPC invocation

coordinating process at the end of the parallel section and make its provisional changes to the protocol or other state permanent. However, this processing is not on the critical path, since it is in parallel with the application activity. Indeed, as much of the cost as possible should be moved into this housekeeping category, or performed speculatively before the next communication is received.

The experiments to test this architectural approach needed a flexible parallel processing environment. A small network of Transputers was used, because this allowed decisions on the resourcing of different processes to be changed on the basis of the preliminary results obtained. It also provided a familiar programming environment for system development. The host workstations used were SUN SPARCstation 1s. In any extended project, absolute performance is likely to be maximized by exploiting the faster hardware becoming available with the passage of time. However, this investigation needed a great deal of detailed performance measurement, and these rather elderly machines were retained because they could be dedicated to it. Absolute performance is, in any case, not the primary aim of the work; rather, the aim has been to compare the performance of the various architectural alternatives, on similar hardware. The conclusions are then just as valid for faster hardware, as long as relative speeds are preserved.

The structure of this paper reflects the phases of the investigation. Section 2 of the paper examines the distribution of RPC cost between the architectural components found in the baseline system in its unmodified form. Section 3 describes the restructuring of the system to place the bulk of the communications support activity in the parallel processing network front end, and section 4 looks at the specific problems of transferring as much as possible of the marshalling and unmarshalling work to this interface. Section 5 draws architectural conclusions.

The basic principles set out here could be applied to a number of communication problems. High performance RPC systems are likely to form the basis of the whole of the next generation of distributed systems, and this work has enabled the design of a new range of high performance intelligent interfaces capable of supporting the open interconnection of systems with different internal architectures.

\section{BASELINE}

Initial performance measurements were made using the unmodified ANSAware platform running over SunOS 4.1.3. The ANSA Echo test is provided as a test service for ANSA's Remote 


\begin{tabular}{ll}
\hline$\%$ execution & \multicolumn{1}{c}{ function } \\
\hline 26.6 & sendto() \\
25.0 & remote execution \\
15.2 & select() \\
11.2 & marshal / unmarshal \\
8.8 & recvfrom() \\
6.5 & schedule \\
6.5 & memory \\
0.2 & application \\
\hline
\end{tabular}

Table 1 Profiling analysis of the echo test

Procedure Call mechanism. It consists of two capsules, the client and server. The client was modified to provide a non-interactive process which executes a set number of RPCs and then exits after printing the total execution time. The server echoes the data contents of the RPC as its result.

\section{Profiling}

Profiling was performed using the standard profiling options in both the capsules and the kernel. The kernel was built using the $-\mathrm{p}$ option to config (8). Once the kernel has been booted, statistics gathering is handled by kgmon ( 8 ), which allows profiling to be started, stopped and the profiling buffers to be reset, so that there can be multiple tests without rebooting the machine. The data gathered is processed by gprof (1). The overhead of running a profiled kernel is that the system spends $5-25 \%$ of its time in the profiling code; there is thus a notable degradation over a non-profiled system.

Analysis of this profiling information for RPCs whose payload is a null string is shown in Table 1. Both client and server exhibit similar timing breakdowns; the server is shown here.

The time required to execute the RPC is dominated by the system functions. It should be noted that the marshalling code takes a significant amount of time considering the size of the payload; this is because call information such as the name of the operation requested and a dispatcher key must be marshalled. (See section 4). A new interface technology must minimise both the amount of time spent in system functions and the time required to marshall the payload. Futher detailed ANSAware profiling information can be found in [Abeysekera, 1993].

\section{CONNECTING THE FRONT END}

The RPC network front end processor is connected to a Unix host via the SCSI bus (see Figure 3 ). The front end comprises a small network of transputers mounted in a PC which provides program loading, control and monitoring.

Specialised Transputer modules (TRAMs) act as a SCSI processor device and an ethernet interface. Up to four general purpose TRAMs are available to carry out computational tasks. A new kernel device driver connects the Unix environment on the SPARCstation to the TRAMs. 


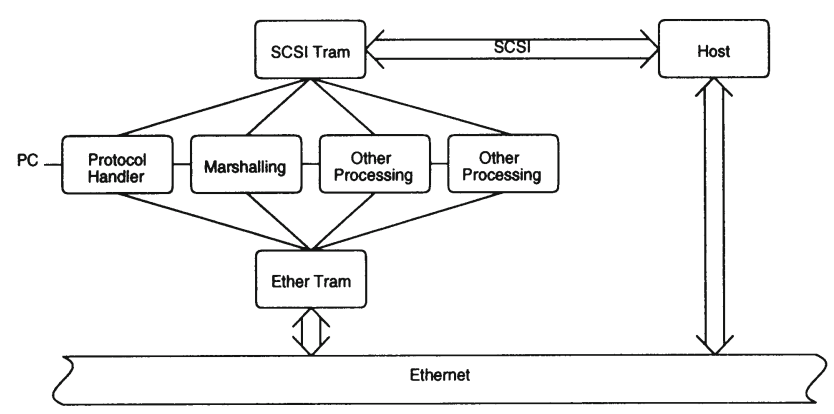

Figure 3 The RPC sub-system

ANSAware has been modified to move various functions into the front end, accessed by the new driver interface.

SCSI was chosen because it is a general purpose bus available on a wide selection of workstations. It thus gives the front end a degree of portability. A change in host would require some recoding of the kernel module but the structure adopted should keep this to a minimum (see section 3.2). TRAMs were adopted for the front end because they provide a flexible embedded parallel processing environment with proven support tools.

\subsection{The ANSAware structure}

The result of building an ANSAware application is a capsule with combines user code and a variety of support functions into a single operating system process. The key components of a capsule are shown in figure 4. The interpreter, execution protocol and message passing service are built into a library. The marshalling stubs are generated by a stub compiler and are compiled with the application; we shall examine marshalling further in section 4.

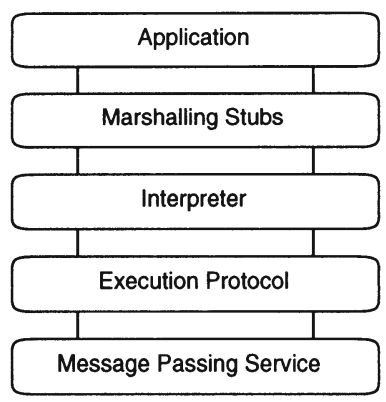

Figure 4 Structure of an ANSAware capsule

\section{Interpreter}

The interpreter provides a distributed abstract machine supporting the application. It manages a multi-threaded environment and supports communication. A channel is an abstraction of a 
communication mechanism between a client and server. An endpoint of a channel is refered to as either a plug or socket: a plug is associated with the client, a socket with the server. A session represents use of a channel by a particular thread, managing state information, local to the capsule, about a remote interaction. Some of this state information, for example the Execution protocol / Message Passing Service pair used, will be fixed when the session is created. The Client and Server maintain separate session information. However, repeated RPCs will re-use an available session.

\section{Execution Protocol}

An execution protocol implements a strategy for communication between capsules. This work concentrated on the Remote Execution protocol (REX), provided as part of the ANSAware 4.1 release, which supports reliable peer to peer operations. The execution protocol is responsible for the reliable delivery of messages via a supporting message passing service. An execution protocol must maintain the session table entry for the current interaction. All execution protocols are accessed through a table called the protocol map, which provides dynamic selection at runtime however, it requires all state information for the invocation to be held in global structures.

\section{Message Passing Service}

A message passing service (MPS) manages low level communication between two host machines. It is responsible for the transmission and receiption of messages and connection to, and disconnection from, the remote host. There is no assurance of quality of service; all MPS are regarded as unreliable.

\subsection{The Unix structure}

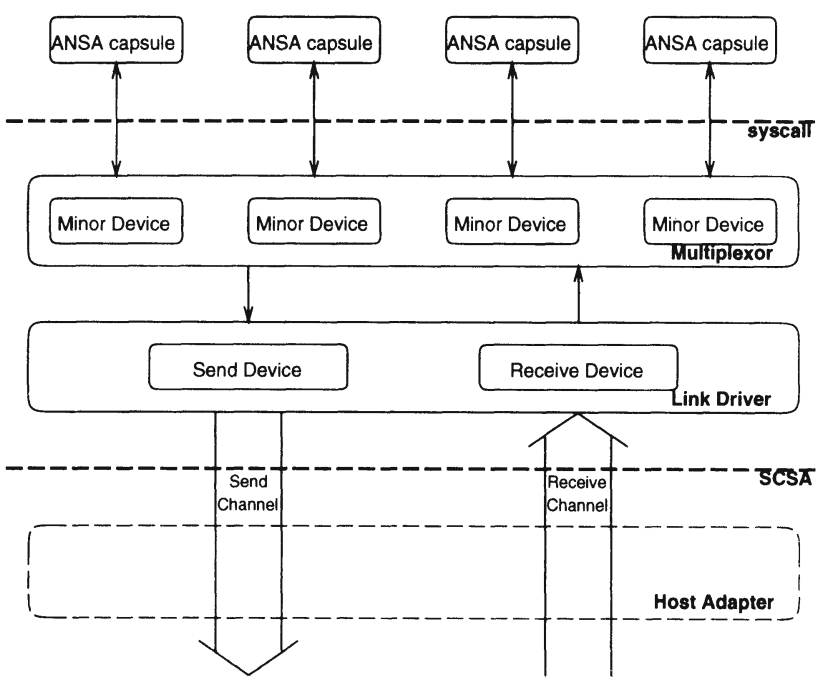

Figure 5 The Kernel Module Structure 
Support for the front end is provided by a new kernel module [Penny, 1994] with two distinct tasks: the first is to provide multiple RPC interfaces to the application enviroment, the second is to communicate via the SCSI processor with the implementation on the TRAMs. These tasks are accomplished by using two device drivers: a multiplexor and a link driver. This division of tasks is analagous to the relationship between the file system and disk device driver, and yields both functional separation and portability.

\section{The Multiplexor}

The multiplexor is a classical pseudo-device in Unix terms. It provides a number of characterspecial devices, each one offering an autonomous RPC slot. It multiplexes these interfaces onto the link provided by the link driver. The multiplexor itself knows nothing about SCSI.

\section{The Link Driver}

The Link Driver supports an asynchronous send / receive interface across the SCSI bus to the TRAMs, providing reliable delivery of commands to the TRAM. The multiplexor is informed only when writes complete or network input has arrived. The driver communicates with the SCSI host adapter via the Sun Common SCSI Architecture.

The SPARCstation acts as the SCSI initiator and the TRAMs implement the functionality of a SCSI processor device. The TRAMs must be able to interrupt the host; polling is not acceptable for a low latency interface. To implement this model the TRAMs are required to provide a pair of processor devices [Smith, 1994]. Although both devices offer a send/receive interface, they are regarded as channels to a single device, one being used as a send channel and the other as a receive channel. The link driver sees the two processor devices as attached at the same SCSI target identifier but with different logical unit numbers. The host will emit a receive to the receive channel whenever it has the resources required, and if the TRAMs have data for the host it will be returned, otherwise they will disconnect and reconnect later when data becomes available. The host will issue a send whenever it has data available.

\section{Optimizations}

It was originally the intention to use direct memory access (DMA) to copy all payload data directly from the users data area to the TRAMs. This requires the relevant pages of the user process memory to be locked down. Detailed timing analysis of the multiplexor revealed that locking and unlocking the users pages to enable DMA takes a significant amount of time, 300 and $185 \mu$ seconds respectively. Because the host adapter used cannot perform any kind of scatter-gather I/O this strategy requires two SCSI transactions, a costly prospect for small payloads.

For small payloads it is faster to copy the data into a kernel buffer which is contiguous with the command block and hence can be transferred in a single SCSI transaction. A SPARC1+ can copy approx 10 MBytes / second from the capsule to the kernel buffer. Thus a mixed strategy is adopted, in which small transfers involve a copy, but long transfers use direct-DMA. In order to ensure a smooth transition between the strategies, and fit the buffer into a multiple of the host pagesize, the transition point is set at 12 Kbytes.

Another bottleneck found during timing analysis is the allocation and freeing of DMA resources for the transmission of commands across the SCSI bus. The buffering strategy used allows the drivers to control where in memory the buffers are allocated. By ensuring that they are allocated in memory visible to the DMA engine, the time required to allocate DMA resources is cut from approx $130 \mu$ seconds to $30 \mu$ seconds. 


\section{Trex}

ANSAware support for the front end is provided by a new execution protocol implementation called Trex. On the host machine this involves function stubs which exchange information across the SCSI bus to the transputers. This "execution protocol" supports the full protocol interface; however the actual execution protocol, session handler etc, are implemented within the TRAMs [Smith et al., 1995]. The binding functionality of ANSAware was changed to make Trex the default execution protocol on systems which supported it.

\subsection{The Transputer structure}

The front end is designed to provide a basic support structure, with SCSI and ethernet, and a flexible processing enviroment. The processing environment allows the movement of functionality between processors to examine the performance of different program structures.

\section{Operation of the Transputer Subsystem}

The first notification the Transputer system has of an RPC call is when the host connects and transfers the RPC arguments to the SCSI TRAM. The SCSI TRAM acts wholly as a buffered link interface between the host and Trex processor; no processing of the data is undertaken on it. All SCSI transactions are handled by a microprogrammable NCR 53C710 SCSI processor on the SCSI TRAM, and the Transputer is interrupted only when it has data from the host or it is able to send further data to the host.

The Trex processor transmits packets either on reception of an RPC call from the host, retransmission of a packet for which no acknowledgement has been received, or transmission of a fragmented packet. When one of these events occurs the corresponding session entry is located from the session table (if it exists, otherwise it is reconstructed), the state updated, and a Trex header is built. The data is then appended to the header and the packet sent to the ethernet processor for transmission to the network. The Trex processor can also be invoked on reception of a packet from the network, which can either update the state of the session entry (such as receipt of packet acknowledgements), or return data to the host. If data is to be returned to the host, a host header is prepended to the data to enable the ANSA kernel to locate the corresponding user session and buffers in which to place the data.

The building and decoding of the IP ethernet frames is managed entirely by the ethernet TRAM. Data which requires transmission to a remote host is received on a Transputer link from one of the other TRAMs and placed at the correct offset in a prototype packet. Other necessary fields which are required to send the packet (such as IP addresses and UDP port numbers) are received with the data and assigned to the correct fields. The ethernet destination address is acquired from a database of IP/ethernet address pairs and the packet transmitted. If no such pair can be found the packet is saved and an ARP request for the address is placed onto the network. When a host replies with the required address, the address is cached in the database, and the packet is transmitted.

Reception of a packet proceeds in a reverse fashion. If the packet fails to the meet the requirements of the protocol specification at any stage, it is discarded. If the UDP port number is in an acceptable range, the UDP packet data and address information are passed onto the Trex processor. Figure 6 shows how the SCSI, Trex and network process are integrated in the Transputer system.

There are two levels of parallelism within this basic system. First the Transputer system 


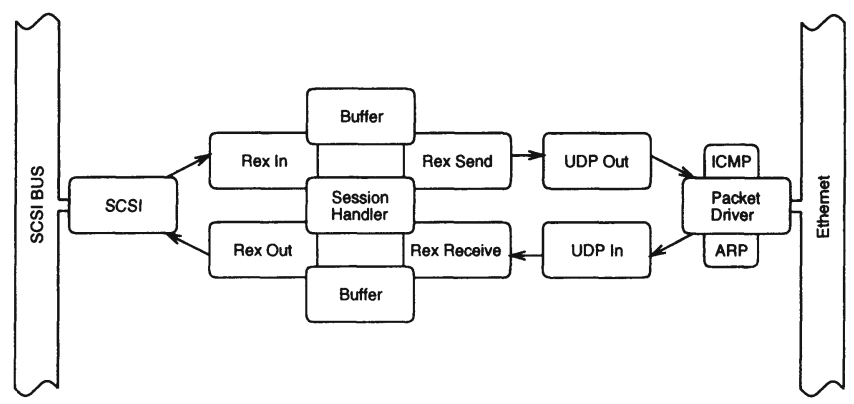

Figure 6 occam process structure

operates in parallel with the host processor. Once the RPC call has been received by the Transputer system the host is free to perform any house-keeping functions and to process other tasks whilst the Trex protocol machine is progressing. Although this provides additional host processor time it does nothing to reduce the latency of the processing of the current Trex packet. The parallelism within the Transputer system is pipelined and allows the tasks of the SCSI, Trex, and network processors to proceed in parallel. With the removal of the Trex session handler from the host kernel and its execution within the Transputer system, a reduction in execution time is obtained due to the lower system overhead and the more efficient instruction execution of the Transputer. It is also noted that the procéssing of packet acknowledgements and fragmentation control is performed without host processor intervention, thus reducing the execution time of the subsequent user code after the RPC call has terminated.

\section{Parallel Refinement of the REX Protocol}

Performance measurements of the packet construction revealed that there was some reduction in latency from implementing the REX session handler and packet construction in the Transputers. Analysis of both the UDP and REX protocols showed new areas for parallelisation. Taking a global view of the flow of data through the protocol stacks reveiled that the processing of protocol headers could be performed in parallel and that only a small subset of the packet headers relied upon the data being sent.

On transmission, inspection of the format of the UDP datagram showed that only eight (out of a total of eighteen) fields of the UDP datagram were dependant on data held by the REX session table. The fields dependent on the REX packet were the datagram sizes, the source and destination UDP port numbers, and the destination IP addresses. The datagram headers and remaining fields could be built whilst the REX session handler was processing the packet, thus saving a significant amount of time. The IP and UDP checksum fields could also be part computed during this time with the remaining values applied when the data had arrived from the REX processor. This optimisation reduced the overhead of building the UDP and IP packet headers by $20 \mu$ seconds.

Incoming data offered less scope for parallel processing as the majority of the processing of the packet headers was required in order to decide whether the packet was to be passed from the network to the REX processor. The only significant optimisation obtained was in the calculation of the packet checksums which could be performed concurrently with the REX 


\begin{tabular}{rrl}
\hline initial & optimised & \multicolumn{1}{c}{ Module } \\
\hline $40.1 \%$ & $48.5 \%$ & SCSI processor \\
$31.3 \%$ & $23.9 \%$ & multiplexor \\
$16.6 \%$ & $20.0 \%$ & link driver \\
$12.0 \%$ & $7.6 \%$ & SCSA library routines \\
\hline $100.0 \%$ & $82.0 \%$ & Relative Execution Time \\
\hline
\end{tabular}

Table 2 Timing analysis of the kernel module

session processing. A successful checksum calculation would result in the commitment of the change of REX session state and initiation of a SCSI transfer of the data to the host.

\subsection{Performance}

Timing analysis of the kernel module and application support uses an event timing mechanism written as part of the project. When a timing point is encountered an event identifier (16 bits), corresponding state information (32 bits), and timestamp (struct timeval) are stored in a data area set aside as a timing buffer. The kernel and application use different versions of the mechanism but share the data format to enable use of a common suite of analysis tools.

\subsection{Execution Protocol Analysis}

Because there are no formal parameters in the protocol interface, the execution protocol must gather all the necessary data from global structures. The Trex function stubs spend most of their time gathering this information before they can initiate a call to the drivers.

The function stubs do not set any timers in the ANSAware nucleus. All retries and ACK's are done by the TRAMs. This means that once a call has been sent no processor time will be used on behalf of the calling thread until the reply is received.

\section{Kernel Analysis}

The majority of execution time in the kernel module is spent in the SCSI communication to the TRAMs. Table 2 gives a percentage breakdown of the time spent in each part of the kernel module.

The optimizations mentioned in section 3.2 saved $18 \%$ overall CPU time but do not alter the ordering of components in the timing analysis. One major overhead is that, unlike the SCSI TRAM, the SPARC1 SCSI processor, an NCR53C90, is not $\mu$ programmable. This means that all interrupt handling and state transition must be performed by the host adapter driver in the host operating system.

\section{Deferral of Activity}

To reduce communication latency it is desirable to move as many tasks as possible off the critical path, whether this be by performing them at configuration time or moving them into CPU "idle" time. Two such optimizations have been identified in the host operating system. 
1. The link driver pre-allocates a number of SCSI packets for both the send and receive devices when it is initialised. Because these packets can be re-used this moves their allocation off the critical path.

2. The pending receive command held by the TRAMs must be renewed each time the TRAMs interrupt the host because the resources associated with it are used. However, this is an expensive action, and is not needed until further data is to be transferred from the front end. It is possible to initiate this renewal when a minor device blocks on a read select, ie. a capsule wishes to read data but none is available. This means that the front end cannot interrupt the host whilst a capsule processes a call or reply, which is, perhaps, not an undesirable side effect. If multiple capsules are blocked awaiting network traffic there is no option but to renew the command on the critical path.

\section{MARSHALLING}

As the size of the payload increases, marshalling comes to dominate the time required to perform an RPC (see figure 7). The marshalling process can be broken down into two sections, the gathering of data dispersed across the address space of a capsule and the conversion of that data into a format suitable for transmission.

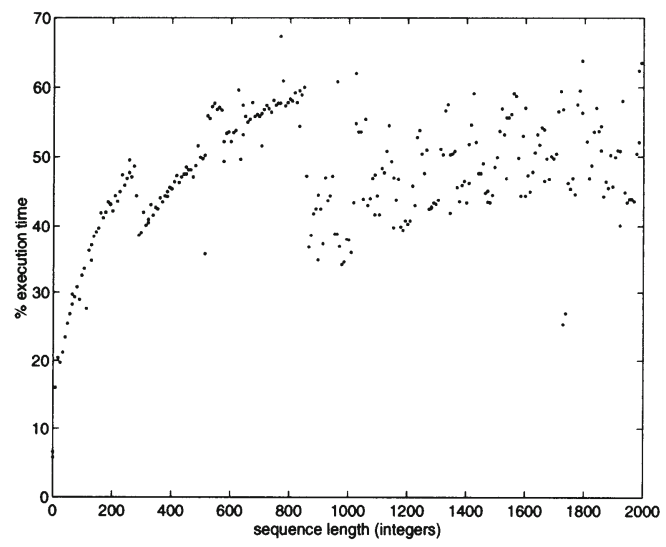

Figure 7 Marshalling Costs of SEQUENCE OF INTEGER

Marshalling code for an ANSAware capsule is constructed by the stub compiler (stubc) based upon the Interface Definition Language (IDL) specification of the capsule interface. IDL supports twelve basic data types and five constructors which are used to build more complex types from the basic types and from other contructed types.

Each operation within an interface has a signature consisting of a name, an argument list and a result list. Both the argument list and result list are expressed in terms of the basic types and constructors. The stub compiler (stubc) compiles these IDL specifications to give $\mathrm{C}$ source files for the client and server stubs and marshalling code which is common to both the client and server. 
Before data from a capsule is transmitted over the network it is copied into a new buffer twice. The first copy is done by the ANSAware marshalling code to provide a contiguous buffer which is used as the payload of a REX packet; format conversion is performed as part of this copy. The second copy is from the capsule's address space into the kernel's address space.

The proposed marshalling strategy minimizes the amount of data copying which is performed by the marshalling process by having it done by the multiplexor minor device. Format conversion is performed on the TRAMs in parallel with the protocol management.

\subsection{Kernel Marshalling}

The kernel marshalling strategy differs from the standard ANSAware marshalling strategy in a number of key ways. Probably the most important is that the stub layer is more closely integrated with the interpreter level. Specifically, the stub layer must use a different marshalling strategy for different execution protocols. In this experiment, we make the assumption that we could implement a rollback mechanism which would alter the marshalling strategy if the primary execution protocol (Trex) fails.

Marshalling proceeds in the following stages :

1. The capsule calls the marshalling function in exactly the same way as normal.

2. The marshalling function, which is generated by a modified stubc, copies an interface descriptor (see below) into the data portion of the ANSAware buffer and extracts an argument frame (see below) from the arguments to the marshalling function.

3. The call then proceeds as normal until it reaches the multiplexor minor device. The multiplexor minor device calls a marshalling module, which is part of the kernel.

4. The marshalling module sequentially parses the interface descriptor, using the argument frame to provide data locations. It copies the data into the kernel buffer immediately after the interface descriptor. Control is then returned to the multiplexor.

A similar strategy is used to unmarshall inbound requests; the capsule must construct an argument frame which contains the data areas into which unmarshalling should occur.

\section{Interface descriptors}

An interface descriptor is a variable length structure which describes the type of each element in an interface operation's argument list. The minor device parses this structure to perform the gathering of data into a contigous kernel buffer. The syntax of an interface descriptor is:

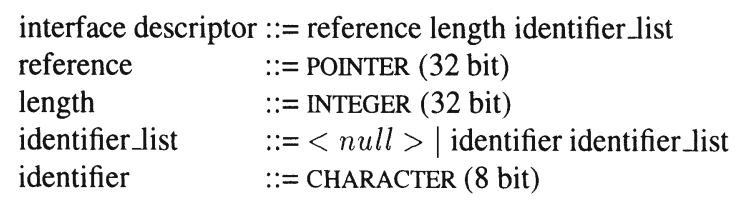

Where reference is the location of the argument frame in memory and length is the number of identifiers in the identifier list. 


\section{Argument frames}

An argument frame contains data and references necessary to marshall an interface in accordance with its interface descriptor. For the basic data types the argument frame will contain the value itself. For constructed types the argument frame will contain a reference to the beginning of the structure in memory. The syntax of an argument frame is:

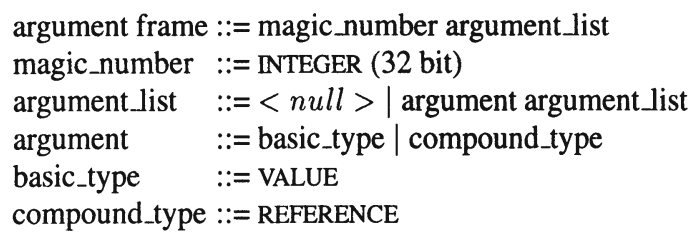

\section{Example}

Consider an interface with the following IDL definition :

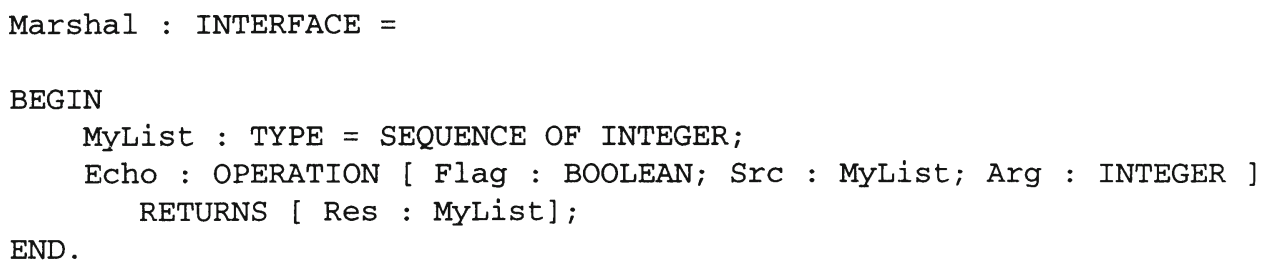

Figure 8 shows an example instance of this interface and the interface descriptor and argument frame which would be produced by stubc.

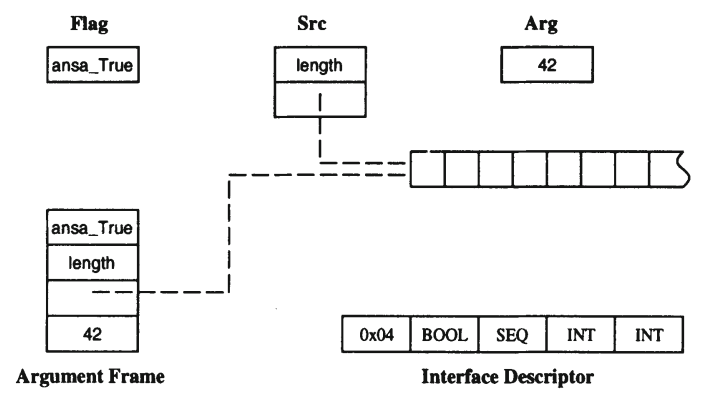

Figure 8 Example marshalling structures.

\section{System Integrity}

If a capsule provides a corrupt argument frame then it is possible that the integrity of the kernel could be compromised. Because the kernel marshalling code uses the argument frame to provide locations in capsule memory it is possible that it could attempt to indirect through an invalid pointer when "pointer chasing". It is not possible to marshall data from kernel memory, nor is 
it possible to marshall data from other processes as they are not in context. A data copy into the kernel which takes a pagefault will cause the error to be propogated back to the capsule.

The situation is alleviated by insisting on a valid magic number at the begining of the argument frame and because all the marshalling code is produced by the stub compiler, stubc. Unlike Sun's XDR library [Sun Microsystems, 1987] there is no application program interface to the ANSA marshalling routines, thus allowing some stronger compile time validation of the code to be performed.

\subsection{Transputer Marshalling}

The Transputer marshalling code is executed in parallel with the REX protocol handling; its primary function is format conversion. The marshalling processor receives the payload of the RPC from the SCSI interface processor and passes the marshalled data to the network processor. Inbound packets are processed in a similar manner but the host transfer can be aborted if the payload does not meet the required format, eg. there is insufficient data.

\section{Buffering}

The transfer of data from the SCSI processor to the marshalling processor is segmented into fixed size buffers, the last segment being scaled to fit any residual data. The same strategy is used for the transfer between the marshalling processor and the network processor. This pipeline allows the marshalling process to overlap format conversion processing with $\mathrm{I} / \mathrm{O}$ processing. The revised structure of the TRAM system is shown in Figure 9.

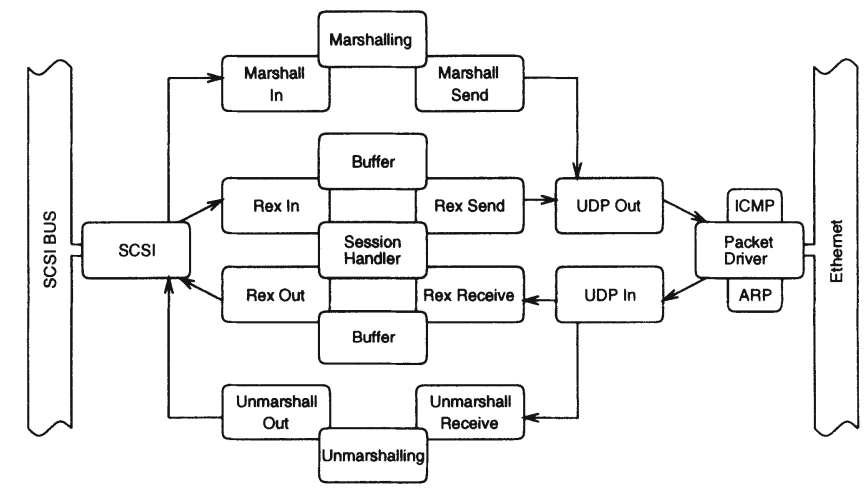

Figure 9 Transputer Marshalling Program Structure

\section{Payload Structure}

The data which the marshalling process receives uses the same interface descriptor (section 4.1) structure as the kernel multiplexor, but the reference to the argument frame is not used. The RPC payload immediately follows the interface descriptor.

The marshalling process must extract a local copy of the interface descriptor so it can complete the parsing after the first segment, containing the original copy, has been freed. The process parses the interface descriptor performing any format conversion on the data in the payload area. Segments are sent to the network processor as soon as they are complete. 


\subsection{Performance}

A modified Echo interface was used to perform timing measurements of the marshalling code. The measurements concentrate on the use of the SEQUENCE OF constructor, an IDL data type consisting of a length field and a reference to a variable length data space.

\section{ANSAware Marshalling}

The time required to marshall a sequence can be broken down into two components. The first is the overhead of the structure itself, which should remain constant across the different element types supported by the constructor. The second component is the cost per element which, for the base types, we would reasonably expect to be proportional to the size of each element.

\section{Kernel Marshalling}

The approach is viable if the time required is less than the standard method for reasonably small numbers of elements within a sequence. Both the length and reference fields of the structure are on the argument frame. The length field must be copied into the data buffer; the reference is used to provide the location of the data in the capsule's address space.

\section{Transputer Marshalling}

If the parameter data is bigger than the buffer size in the marshalling process, a significant amount of processing can be carried on in parallel with data transfer. Measurements have shown that $2.25 \mu$ seconds of processing per integer can be carried out without increasing the total RPC latency, this gives a substantial saving in the time required to perform format conversion.

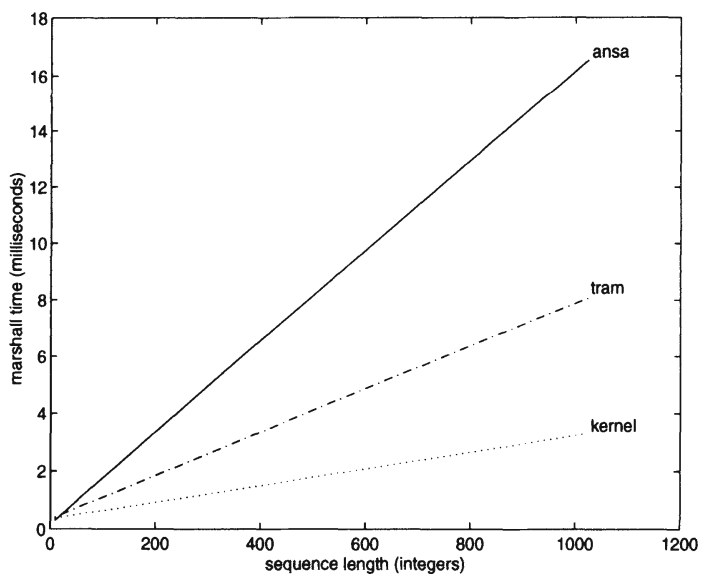

Figure 10 Marshalling SEQUENCE OF INTEGER.

Figure 10 shows the trends, generated from a series of measurements, in the total cost of marshalling, including kernel copying, within the echo test. The combined cost of kernel and transputer marshalling is shown (tram) and the proportion of that time which is directly attributable to the kernel part of the strategy (kernel). 


\section{CONCLUSIONS}

This work has demonstrated that significant performance improvements can be made by applying the optimistic concurrency approach to the implementation of RPC-based communications. Offloading the handling of network and execution protocols reduced the CPU load on the host system significantly, saving $17 \%$ for RPCs with trivial parameters. Transferring the format conversions required for marshalling and unmarshalling of medium sized parameter structures reduced CPU loading, within the marshalling sub-system, by $53 \%$.

The main limitation of the experimental configuration was the high cost of managing SCSI transfers within the UNIX system. If a more efficient inter-processor link had been available, the savings observed would have been even larger. Some $56 \%$ of the time for a trivial RPC can be ascribed to the cost of communication between the workstation and the network front end processor.

In particular, this overhead ruled out management structures in which one piece of network communication resulted in multiple interprocessor transfers. Thus overlap was restricted to transfer initiation, rather than user process selection, loosing part of the potential benefit. A more modern SCSI adaptor would have overcome some of these problems, and increased the performance still further.

If an even lower overhead link were available, such as a shared memory structure, it would be interesting to combine the parallel processing approach demonstrated here with a more intimate access to user data structures or stack contents, transferring information on the interface types to be supported on demand, and moving part of the kernel marshalling cost to the front end.

Despite these hardware limitations, significant performance improvements have been demonstrated, and the general principle of delegating the more costly aspects of protocol processing and message formatting to a front end has been shown to be effective for RPC traffic. Format conversion costs, in particular, can be almost completely hidden. The magnitude of the savings increase steadily as the volume of parameter data is increased, so that the techniques are likely to be particularly effective in application areas where RPC parameter sets are large, such as database access and multimedia support.

The performance increase expected from this technique would be maintained if the speed of the various processors, interfaces and communications paths were increased in the same proportion. Here again, it is the speed of inter-process communication (and associated cache constraints) which is likely to be the main limitation as the host processors become progressively more sophisticated.

Finally, although the current work is specific to the internal structure of ANSAware, the same techniques should be applicable to other RPC systems of similar functionality. It should be possible, for example, to apply them to well engineered ORB implementations.

The basic principles set out here could be applied to a number of communication problems. High performance RPC systems are likely to form the basis of the whole of the next generation of distributed systems, and this work has enabled the design of a new range of high performance intelligent interfaces capable of supporting the open interconnection of systems with different internal architectures.

The authors would like to acknowledge their appreciation of the support provided for this work by the UK EPSRC, under grant number GR/H/44684. 


\section{REFERENCES}

[Abeysekera, 1993] Abeysekera, D. C. R. (1993). Performance of Thread Based Distributed Systems. PhD thesis, University of Kent, UK.

[ANSA, 1989] ANSA (1989). ANSA Reference Manual, Release 01.00. APM Ltd, Cambridge, UK.

[ISO, 1995] ISO (1995). Basic reference model of open distributed processing. ISO 10746 (parts 2 and 3).

[Linington, 1995] Linington, P. (1995). RM-ODP - the architecture. In Proceedings of ICODP'95.

[OMG, 1991] (1991). Common Object Request Broker: Architecture and Specification, OMG document 91.12 .1 revision 1.1.

[Penny, 1994] Penny, I. A. (1994). The Design and Implementation of the RPC Device Drivers. Technical Report 5/94, Computing Laboratory, University of Kent, UK.

[Rozier, 1986] Rozier, M. and Martins, J. L. (1986). The Chorus Distributed Operating System: Some design issues, pages 261-287. Springer-Verlag.

[Schroeder, 1990] Schroeder, M. D. and Burrows, M. (1990). Performance of firefly RPC. ACM TOCS, 8(1):1-17.

[Smith, 1994] Smith, A. B. (1994). Implementing a transputer SCSI interface. Technical Report 22/94, Computing Laboratory, University of Kent, UK.

[Smith et al., 1995] Smith, A. B., Ibbetson, A. L., Linington, P. F., Penny, I. A., and Tripp, G. E. W. (1995). A parallel implementation of the ANSA REX protocol. In Proceedings of WTC'95.

[Sun Microsystems, 1987] Sun Microsystems, Inc. (1987). XDR : External data representation standard. ARPA Network Information Center RFC1014.

[van Renesse, 1988] van Renesse, R., van Staveren, H., and Tanenbaum, A. (1988). Performance of the world's fastest distributed operating system. Operating Systems Review, 22(4):25-34. 